\title{
RING COUPLING-BASED COLLABORATIVE FAULT-TOLERANT CONTROL FOR MULTI-ROBOT ACTUATOR FAULT
}

\author{
Jing He, ${ }^{*, * *}$ Lin $\mathrm{Mi},{ }^{*}$ Jianhua Liu, ${ }^{*}$ Xiang Cheng, ${ }^{*}$ Zhenzhen Lin, ${ }^{*}$ and Changfan Zhang*
}

\begin{abstract}
In this paper, a ring coupling-based fault-tolerant control scheme is proposed to synchronize multi-robot systems with actuator faults. The control scheme includes a sliding-mode control law and a slidingmode observer. The sliding-mode control law is given via the ring coupling strategy for a multi-robot system. To observe the status of unmeasurable variables and unknown fault control information, a sliding mode observer is designed such that the unknown fault information is accurately reconstructed by using the equivalent principle of sliding-mode variable structure. Based on the observed status value and fault reconstructed value, the sliding-mode control law can be adjusted online and thus the collaborative fault-tolerant control is realized online. Finally, simulations and experiments are given to demonstrate the effectiveness of the proposed method.
\end{abstract}

\section{Key Words}

Multi-robot system, actuator fault, ring coupling strategy, fault reconstruction, fault-tolerant control

\section{Introduction}

In recent years, multi-robot systems have been widely used in modern industrial systems, in which high requirements have also been proposed for their synchronization performance. Thus, many scholars have conducted extensive research and have made great progress on studying the problem of synchronous control of multiple controlled object systems [1]-[8]. Khalili et al. proposed a distributed adaptive fault-tolerant leader-following consensus control scheme [9]. Ai et al. developed a master-slave control algorithm and applied it on minimally invasive surgical robots to solve the workspace mismatch problem between

\footnotetext{
* College of Electrical and Information Engineering, Hunan University of Technology, Zhuzhou, Hunan 412007, China; e-mail: hejing@263.net, 1058981366@qq.com, jhliu0615@163.com, chengxiang@stu.hut.edu.cn, ganzhouzhenzhenlin@qq.com, zhangchangfan@263.net

** Hui Lin Packing Dongguan Co., Ltd., Dongguan, Guangdong 523000, China

Corresponding author: Jianhua Liu

Recommended by Prof. Anmin Zhu
}

(DOI: 10.2316/Journal.206.2018.6.206-5487) the master hand and slave arm. However, this work did not consider the effects of unknown input disturbances [10]. In addition, Chen et al. indicated that the anti-jamming capacity of master-slave control strategy is not ideal [11]. Andert et al. applied the virtual shaft synchronous control strategy to the control of vehicle power system, which reduces the speed deviation and improves the synchronization accuracy; however, the influences of faults were not considered [12]. Liu et al. used the hybrid control scheme to achieve the consensus and multi-tracking for dynamical networks [13]-[15]. For the control problems of micro-nanotechnology-related equipment, Ulu et al. proposed a modular control method for cross-coupled control with combining the iterative learning control to improve system tracking accuracy [16]. But, the complexity of the cross-coupled control algorithm has considerably increased with the increase in number of controlled objects [17]. Shi et al. applied the relative coupling control strategy to multi-motor and multi-channel synchronous control, which shows a good synchronization performance [18]-[20]. However, as the number of controlled object increases, the complexity of the system control structure also increases [2]. Moreover, the fault information was not investigated in [16] and [18]-[20]. Compared with the four synchronous control strategies, namely, master-slave control, virtual shaft synchronous control, cross-coupled control, and relative coupling control, the ring coupling control strategy has greater robustness and is thus suitable for systems with multi-controlled objects [21]. Moreover, a control method based on ring coupling control and adaptive sliding-mode control was proposed by $\mathrm{Li}$ et al. for multi-motor speed synchronous tracking control, which ensures the convergence of the velocity tracking error and synchronization error to zero. However, the influence of fault was also not considered [22].

In this paper, by the spirit of the work by Li et al. [22], a collaborative fault-tolerant control method is proposed for multi-robot systems with actuator faults. First, the fault-tolerant control law based on ring coupling control strategy is proposed. An observer is designed to observe the status of unmeasurable variables and unknown fault information. Then, the fault reconstruction method is used to reconstruct the faults precisely. Finally, the observed 
status value and fault reconstructed value are applied to the online adjustment of the control law.

The remainder of this paper is organized as follows. Section 2 introduces the faulty multi-robot system model. Section 3 presents the design of system fault-tolerant control rate. Section 4 describes the design of the sliding-mode observer. Section 5 presents the reconstruction of the actuator fault. Section 6 presents the online adjustment of fault-tolerant control rate. Section 7 gives the simulations with explanations. Finally, conclusion is presented in Section 8.

\section{System Description}

Assuming that the multi-robot system has $n$ robots, the mathematical model of the actuator fault in the $i$ th $(i=1, \ldots, n)$ robot is as follows:

$$
M\left(q_{i}\right) \ddot{q}_{i}+B\left(q_{i}, \dot{q}_{i}\right) \dot{q}_{i}+G\left(q_{i}\right)=\tau_{i}+d_{i}+f_{a i}
$$

where $q_{i}$ is the joint angular displacement; $M\left(q_{i}\right)$ is the robotic inertial matrix; $B\left(q_{i}, \dot{q}_{i}\right)$ denotes the centrifugal force and Coriolis force; $G\left(q_{i}\right)$ is the gravity term; $\tau_{i}$ is the control torque; $d_{i}$ is the unknown bounded function, which represents the unknown input disturbance or system uncertainty; and $f_{a i}$ denotes the actuator fault, which is an unknown bounded function.

There are three main types of actuator failures, namely, stuck, constant gain variation, and constant deviation failure. The fault models can be described as, respectively, $f_{a i}(t)=u_{s i} \quad\left(u_{s i}\right.$ as constant $), \quad f_{a i}(t)=\rho_{i}(t) v_{i}(t)$ $\left(v_{i}(t)\right.$ is controller output, $\rho_{i}(t)$ is the coefficient of constant gain variation), $f_{a i}(t)=v_{i}(t)+\Delta_{i}\left(\Delta_{i}\right.$ is a constant $)$, and $i=1, \ldots, n$. The three kinds of actuator failure models can be described as follows:

$$
f_{a i}(t)=k_{1 i} u_{s i}+k_{2 i} \rho_{i}(t) v_{i}(t)+k_{3 i} \Delta_{i}, i=1, \cdots, n
$$

where $k_{1 i}, k_{2 i}, k_{3 i}=0$ or 1 .

$F_{a i}=d_{i}+f_{a i}$ is defined as the equivalent actuator fault. Thus, (1) can be rewritten as

$$
M\left(q_{i}\right) \ddot{q}_{i}+B\left(q_{i}, \dot{q}_{i}\right) \dot{q}_{i}+G\left(q_{i}\right)=\tau_{i}+F_{a i}
$$

For a one-DOF (degree of freedom) manipulator, $M\left(q_{i}\right)$ and $B\left(q_{i}, \dot{q}_{i}\right)$ are both constants. Moreover, $G\left(q_{i}\right)=m g l \cos q_{i}$. Thus, (3) can be rewritten as

$$
M_{i} \ddot{q}_{i}+B_{i} \dot{q}_{i}+m g l \cos q_{i}=\tau_{i}+F_{a i}
$$

\section{Design of Collaborative Fault-tolerant Control Rate Based on Ring Coupling}

The position-tracking error $e_{i}$ of the $i$ th robot is defined as

$$
e_{i}=q_{d}-q_{i}
$$

where $q_{d}$ is the given input position signal.
The position synchronization error $\varepsilon_{i}$ of the $i$ th robot and the $(i+1)$ th robot is

$$
\varepsilon_{i}=q_{i}-q_{i+1}
$$

where $i=n, q_{i+1}=q_{1}$.

The tracking error $E_{i}$ in the $i$ th robot control system by using the ring coupling compensation is

$$
E_{i}=e_{i}-k_{i} \varepsilon_{i}
$$

where $k_{i}$ is a positive constant that needs to be designed.

A nominal system has no equivalent actuator fault, that is $F_{a i}(t)=0$. An appropriate nominal control law $\tau_{i 0}$ is used for a progressive and stabilization of the system. When the equivalent actuator fault occurs, that is $F_{a i}(t) \neq 0$, an additional control law $\tau_{i f}$ may be designed to realize fault-tolerant adjustment. Thus, for the faulty system (4), the fault-tolerant control law $\tau_{i}$ consists of the nominal control law $\tau_{i 0}$ and the additional control law $\tau_{i f}$, which is shown as follows:

$$
\tau_{i}=\tau_{i 0}+\tau_{i f}
$$

Sliding-mode surface $s$ is selected as

$$
s_{i}=\dot{E}_{i}+c_{i} E_{i}
$$

where $c_{i}$ is a positive constant. Therefore,

$$
\begin{aligned}
\dot{s}_{i}= & \ddot{E}_{i}+c_{i} \dot{E}_{i} \\
= & c_{i} \dot{E}_{i}+\ddot{q}_{d}-M_{i}^{-1}\left(\tau_{i}-B_{i} \dot{q}_{i}-m g l \cos q_{i}+F_{a i}\right) \\
& -k_{i} \ddot{\varepsilon}_{i}
\end{aligned}
$$

In the sliding-mode surface which is $s_{i}=0$ near the origin, the following condition should be satisfied to ensure the existence of the sliding-mode area of the system:

$$
s_{i} \dot{s}_{i} \leq 0
$$

Thus,

$$
\begin{gathered}
s_{i} \dot{s}_{i}=s_{i}\left[c_{i} \dot{E}_{i}+\ddot{q}_{d}-M^{-1}\left(\tau_{i}-B \dot{q}_{i}-m g l \cos q_{i}+F_{a i}\right)\right. \\
\left.-k_{i} \ddot{\varepsilon}_{i}\right] \leq 0
\end{gathered}
$$

The control law $\tau_{i}$ based on reaching law is set as

$$
\begin{aligned}
\tau_{i}= & M\left[c_{i} \dot{E}_{i}+\ddot{q}_{d}-k_{i} \ddot{\varepsilon}_{i}+a s+b \operatorname{sgn}\left(s_{i}\right)\right] \\
& +B \dot{q}_{i}+m g l \cos q_{i}-F_{a i}
\end{aligned}
$$

where $a$ and $b$ are constants to be determined later. When the fault does not occur (i.e., $F_{a i}=0$ ), the control law $\tau_{i 0}$ of the nominal system is

$$
\begin{aligned}
\tau_{i 0}= & M\left[c_{i} \dot{E}_{i}+\ddot{q}_{d}-k_{i} \ddot{\varepsilon}_{i}+a s+b \operatorname{sgn}(s)\right] \\
& +B \dot{q}_{i}+m g l \cos q_{i}
\end{aligned}
$$

Otherwise, $F_{a i} \neq 0$, the additional control law $\tau_{i f}$ is

$$
\tau_{i f}=-F_{a i}
$$


Theorem 1. For the robot system described by Equation (4), the designed tolerant control law is shown in (12). When $a>0$ and $b>0$, the system satisfies the stability condition of the sliding mode.

Proof: Let Lyapunov function be

$$
V_{i}=\frac{1}{2} s_{i}^{2}
$$

The derivative of (15) yields

$$
\begin{gathered}
\dot{V}_{i}=s_{i} \dot{s}_{i}=s\left[c_{i} \dot{E}_{i}+\ddot{q}_{d}-M^{-1}\left(\tau_{i}-B \dot{q}_{i}-m g l \cos q_{i}+F_{a i}\right)\right. \\
\left.-k_{i} \ddot{\varepsilon}_{i}\right]
\end{gathered}
$$

Substituting (12) into (16) yields

$$
\dot{V}_{i}=s_{i} \dot{s}_{i}=-a s_{i}^{2}-b\left|s_{i}\right|
$$

When $a>0$ and $b>0$, the following can be obtained as

$$
s_{i} \dot{s}_{i} \leq 0
$$

Thus, the system satisfies the existence and reach ability conditions of a sliding mode, that is, the system will reach the sliding-mode surface $s_{i}=0$ in a finite time from any initial state. Thus, the proof is complete.

By using Theorem 1 and (9), error $E_{i}$ asymptotically converges to zero.

Using (5) and (6) yields

$$
\begin{aligned}
E_{i} & =e_{i}-k_{i} \varepsilon_{i} \\
& =\left(1+k_{i}\right) e_{i}-k_{i} e_{i+1}
\end{aligned}
$$

Equation (18) can be written as follows:

$\left[\begin{array}{cccccc}1+k_{1} & -k_{1} & \ldots & & \cdots & 0 \\ \vdots & \ddots & & & & \vdots \\ 0 & \ldots & 1+k_{i} & -k_{i} & \ldots & 0 \\ \vdots & & & & \ddots & \vdots \\ -k_{n} & \ldots & & \ldots & 0 & 1+k_{n}\end{array}\right]\left[\begin{array}{c}e_{1} \\ \vdots \\ e_{i} \\ \vdots \\ e_{n}\end{array}\right]=\left[\begin{array}{c}E_{1} \\ \vdots \\ E_{i} \\ \vdots \\ E_{n}\end{array}\right]$

Matrix $A$ is set as

$$
A=\left[\begin{array}{cccccc}
1+k_{1} & -k_{1} & \ldots & & \cdots & 0 \\
\vdots & \ddots & & & & \vdots \\
0 & \ldots & 1+k_{i} & -k_{i} & \cdots & 0 \\
\vdots & & & & \ddots & \vdots \\
-k_{n} & \cdots & & \ldots & 0 & 1+k_{n}
\end{array}\right]
$$

If $1+k_{i} \neq 0$ and $1+k_{n}-\frac{k_{n} !}{\left(1+k_{n-1}\right) !} \neq 0$, that is, $k_{i} \neq-1$, then $k_{i}$ is a positive constant that needs to be designed.
Thus, matrix $A$ is a non-singular matrix. If $E_{i}=\left(1+k_{i}\right) e_{i}-$ $k_{i} e_{i+1}=0$, then $\left[e_{1}, \ldots, e_{n}\right]^{T}$ has only one zero solution; thus, $\varepsilon_{1}=\cdots=\varepsilon_{n}=0$. At this point, the tracking and synchronization errors can be eliminated.

In (12), the designed control law $\tau_{i}$ includes system status variable $\dot{q}_{i}$ and equivalent actuator fault $F_{a i}$, which is usually unknown. Their exact value should be obtained through a certain method. In the next section, a slidingmode observer based on the equivalent actuator fault reconstructed accurately to observe system status; thus, the corresponding observed status value and the reconstructed value of equivalent actuator fault can be acquired and then substituted into the control law $i$ for the online faulttolerant control.

\section{State of the Sliding-Mode Observer and Fault Reconstruction}

System is selected according to (4).

$$
x_{1}=q_{i}, \quad x_{2}=\dot{q}_{i}
$$

The dynamic equation of (4) becomes:

$$
\left\{\begin{array}{l}
\dot{x}_{1}=x_{2} \\
\dot{x}_{2}=M^{-1}\left[\tau_{i}+F_{a i}-B x_{2}-m g l \cos x_{1}\right]
\end{array}\right.
$$

The sliding-mode observer is designed as follows:

$$
\left\{\begin{array}{l}
\dot{\hat{x}}_{1}=\hat{x}_{2}+v_{1} \\
v_{1}=L_{1} \operatorname{sgn}\left(x_{1}-\hat{x}_{1}\right)
\end{array}\right.
$$

$$
\left\{\begin{array}{l}
\dot{\hat{x}}_{2}=M^{-1}\left(\tau_{i}-B \hat{x}_{2}-m g l \cos x_{1}\right)+v_{2} \\
v_{2}=L_{2} \operatorname{sgn}\left(x_{2}-\hat{x}_{2}\right)
\end{array}\right.
$$

where $\hat{x}_{1}$ and $\hat{x}_{2}$ are the observed value of $x_{1}$ and $x_{2}$, respectively; $v_{1}$ and $v_{2}$ are the control terms of the sliding mode; and $L_{1}$ and $L_{2}$ are the positive constants to be designed.

Errors $\hat{e}_{1}$ and $\hat{e}_{2}$ of the observer are defined as

$$
\left\{\begin{array}{l}
\hat{e}_{1}=x_{1}-\hat{x}_{1} \\
\hat{e}_{2}=x_{2}-\hat{x}_{2}
\end{array}\right.
$$

According to (21)-(24), the error equations are

$$
\begin{gathered}
\dot{\hat{e}}_{1}=\hat{e}_{2}-v_{1} \\
\dot{\hat{e}}_{2}=-M^{-1} B\left(x_{2}-\hat{x}_{2}\right)+M^{-1} F_{a i}-v_{2}
\end{gathered}
$$

Considering the error dynamic in (25) and (26), slidingmode surfaces $\hat{s}_{1}$ and $\hat{s}_{2}$ are selected as follows:

$$
\hat{s}_{1}=\hat{e}_{1}, \quad \hat{s}_{2}=\hat{e}_{2}
$$


Theorem 2. If $L_{1}$ and $L_{2}$ are sufficiently large (i.e., satisfying inequalities $L_{1} \geq\left|\hat{e}_{2}\right|+\eta_{1}$ and $L_{2} \geq$ $\left|M^{-1} f_{a i}\right|+\eta_{2}$, where $\eta_{1}$ and $\eta_{2}$ are both positive constants), then the value of the equivalent actuator fault is $F_{a i}=M L_{2} \operatorname{sgn}\left[L_{1} \operatorname{sgn} \hat{e}_{1}\right]$.

Proof: The Lyapunov function is selected as follows:

$$
\hat{V}_{1}=\frac{1}{2} \hat{e}_{1}^{2}
$$

According to (25),

$$
\begin{aligned}
\dot{\hat{V}}_{1} & =\hat{e}_{1} \dot{\hat{e}}_{1}=\hat{e}_{1} \hat{e}_{2}-\hat{e}_{1} v_{1} \\
& =\left|\hat{e}_{1}\right|\left[\left|\hat{e}_{2}\right|-L_{1}\right]
\end{aligned}
$$

When constant $L_{1}$ is sufficiently large, that is, $L_{1} \geq$ $\left|\hat{e}_{2}\right|+\eta_{1}$, then

$$
\dot{\hat{V}}_{1} \leq-\eta_{1}\left|\hat{e}_{1}\right|
$$

As shown in the above demonstration, the system satisfies the existence and reach ability conditions of the sliding mode; that is, within a finite time, the system departing from any initial conditions will reach sliding-mode surface $\hat{s}_{1}=0$. According to the sliding mode equivalent principle [23], when the sliding-mode surface $\hat{s}_{1}$ is reached, $\hat{s}_{1}=\dot{\hat{s}}_{1}=0$.

According to (25),

$$
\hat{e}_{2}=v_{1}=L_{1} \operatorname{sgn} \hat{e}_{1}
$$

The selected Lyapunov function is

$$
\hat{V}_{2}=\frac{1}{2}\left(\hat{e}_{1}^{2}+\hat{e}_{2}^{2}\right)
$$

Thus,

$$
\begin{aligned}
\dot{\hat{V}}_{2} & =\hat{e}_{1} \dot{\hat{e}}_{1}+\hat{e}_{2} \dot{\hat{e}}_{2} \\
& =\dot{V}_{1}+\hat{e}_{2} \dot{\hat{e}}_{2}
\end{aligned}
$$

According to (26),

$$
\begin{aligned}
\hat{e}_{2} \dot{\hat{e}}_{2} & =\hat{e}_{2}\left[-M^{-1} B\left(x_{2}-\hat{x}_{2}\right)+M^{-1} F_{a i}-v_{2}\right] \\
& \leq-M^{-1} B \hat{e}_{2}^{2}+\left|\hat{e}_{2}\right|\left[\left|M^{-1} F_{a i}\right|-L_{2}\right]
\end{aligned}
$$

When constant $L_{2} \geq\left|M^{-1} f_{a i}\right|+\eta_{2}$, then

$$
\begin{aligned}
\hat{e}_{2} \dot{\hat{e}}_{2} \leq & -M^{-1} B \hat{e}_{2}^{2}-\eta_{2}\left|\hat{e}_{2}\right| \\
& \leq-\eta_{2}\left|\hat{e}_{2}\right|
\end{aligned}
$$

Together (29) with (33), we obtain

$$
\dot{\hat{V}}_{2} \leq-\eta_{1}\left|\hat{e}_{1}\right|-\eta_{2}\left|\hat{e}_{2}\right|
$$

Therefore, the system satisfies the existence and reach ability conditions of sliding mode; that is, within a finite time, the system departing from any initial conditions will reach sliding-mode surface $\hat{s}_{2}$. Similarly, when the sliding-mode surface $\hat{s}_{2}$ is reached, $\hat{s}_{2}=\dot{\hat{s}}_{2}=0$.

According to (26) and (30), the value of equivalent actuator fault is

$$
\begin{aligned}
F_{a i} & =M v_{2} \\
& =M L_{2} \operatorname{sgn}\left[L_{1} \operatorname{sgn} \hat{e}_{1}\right]
\end{aligned}
$$

Thus, the proof is complete.

Using the sigmoid function in replacing $\operatorname{sgn}(s)$ to weaken the buffeting of the sliding mode, the reconstructed value of the equivalent actuator fault becomes

$$
\hat{F}_{a i}=M L_{2}\left(\frac{2}{1+e^{-a_{1} L_{1} \operatorname{sgn} \hat{e}_{1}}}-1\right)
$$

where $a_{1}>0$.

\section{Online Adjustment of Fault-tolerant Control Rate}

The online fault-tolerant control for system (4) can be described as follows. The observed value $\hat{x}$ of the system status (i.e., $\hat{q}$ ) and the reconstructed value $\hat{F}_{a i}$ of the equivalent actuator fault are substituted into the control law of (12). The adjusted online fault-tolerant control law $\tau_{i}$ is

$$
\begin{gathered}
\tau_{i}=M\left[c_{i} \dot{E}_{i}+\ddot{q}_{d}-k_{i} \ddot{\varepsilon}_{i}+a s+b \operatorname{sgn}\left(s_{i}\right)\right] \\
+B \dot{\hat{q}}_{i}+m g l \cos q_{i}-\hat{F}_{a i}
\end{gathered}
$$

When the system does not have an equivalent actuator fault $\left[\right.$ i.e., $\left.F_{a i}(t)=0\right]$, the control law $\tau_{i 0}$ (13) of the nominal system is adjusted to

$$
\begin{gathered}
\tau_{i 0}=M\left[c_{i} \dot{E}_{i}+\ddot{q}_{d}-k_{i} \ddot{\varepsilon}_{i}+a s+b \operatorname{sgn}\left(s_{i}\right)\right] \\
+B \dot{\hat{q}}_{i}+m g l \cos q_{i}
\end{gathered}
$$

When the equivalent actuator fault occurs [i.e., $F_{a i}(t) \neq 0$ ], the designed additional control law $\tau_{i f}(14)$ is adjusted to

$$
\tau_{i f}=-\hat{F}_{a i}
$$

Theorem 3. The online fault-tolerant control law designed for the robot system described by (4) is shown in (37). When $b \geq\left\|M^{-1} B L_{1}\right\|+L_{2}$, the system satisfies the stability condition of the sliding mode.

Proof: By selecting the Lyapunov function,

$$
V_{i}^{\prime}=\frac{1}{2} s_{i}^{2}
$$


Considering the derivative of (40) and then substituting it into the adjusted control law (37),

$$
\begin{aligned}
\dot{V}_{i}^{\prime} & =s_{i} \dot{s}_{i} \\
& \leq-a s_{i}^{2}-b\left|s_{i}\right|+\left|s_{i}\right|\left(\left\|M^{-1} B \hat{e}_{2}\right\|+\left\|M^{-1}\left(F_{a i}-\hat{F}_{a i}\right)\right\|\right.
\end{aligned}
$$

According to (27), (32), and (33)

$$
\dot{V}_{i}^{\prime} \leq-a s_{i}^{2}-b\left|s_{i}\right|+\left|s_{i}\right|\left(|| M^{-1} B L_{1} \|+L_{2}\right)
$$

When $b \geq\left\|M^{-1} B L_{1}\right\|+L_{2}$, thus

$$
\dot{V}_{i}^{\prime} \leq-a s_{i}^{2}
$$

The proof is thus complete.

The above analysis shows that the system satisfies the existence and reach ability conditions of the sliding mode; that is, the system departing from any initial state other than the sliding-mode surface $s=0$ will reach the sliding-mode surface within a finite time.

\section{Analysis on Simulation and Experiment Results}

In dynamic (4) of one-DOF manipulator,

$$
\begin{gathered}
M_{i}=\left[M_{1}, M_{2}, M_{3}, M_{4}\right]=\left[\frac{3}{4} m l^{2}, \frac{4}{3} m l^{2}, m l^{2}, \frac{9}{8} m l^{2}\right], \\
B_{i}=2.0, \quad(i=1,2,3,4)
\end{gathered}
$$

Herein, $m=1, l=0.25$, and $g=9.8$. A multi-robot system with four one-DOF manipulators is used. The initial position of the system is $q_{i 0}=\left[\begin{array}{llll}-0.5 & -1.5 & 0.5 & 1\end{array}\right]^{T}$, and the given input position signal is $q_{d}=2 \cos (\pi t)$.

The design parameters are as follows:

$$
\begin{gathered}
k_{i}=1 \\
c_{1}=6, \quad c_{2}=8, \quad c_{3}=12, \quad c_{4}=9 \\
a=3, \quad b=30, \quad L_{1}=5, \quad L_{2}=15 .
\end{gathered}
$$

\subsection{Simulation Results}

The performance of the proposed fault-tolerant control scheme is evaluated with a detailed simulation model using the MATLAB/Simulink R2007b. When no equivalent actuator fault exists in the system (i.e., $F_{a i}=0$ ), it is a nominal system and the control law is $\tau_{i 0}$. In this case, Fig. 1 shows the errors $e_{1}, e_{2}, e_{3}$, and $e_{4}$ between the system states $q_{1}, q_{2}, q_{3}$, and $q_{4}$ of four robots and the given input position signals $q_{d}$. Figure 2 shows the synchronization errors $e_{12}, e_{23}, e_{34}, e_{41}$ of the tracking status between adjacent robots. The simulation results are as follows.

The given input signals can be stably tracked by all four robots, as shown by the tracking error in Fig. 1. This result suggests good tracking effect. Figure 2 shows that the synchronization error of the tracking status between

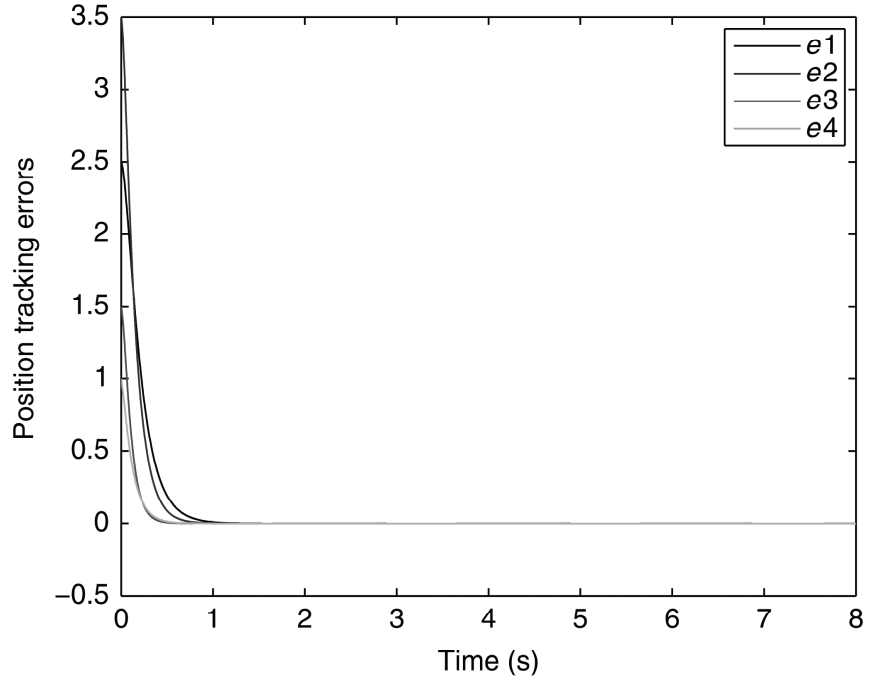

Figure 1. Position-tracking error.

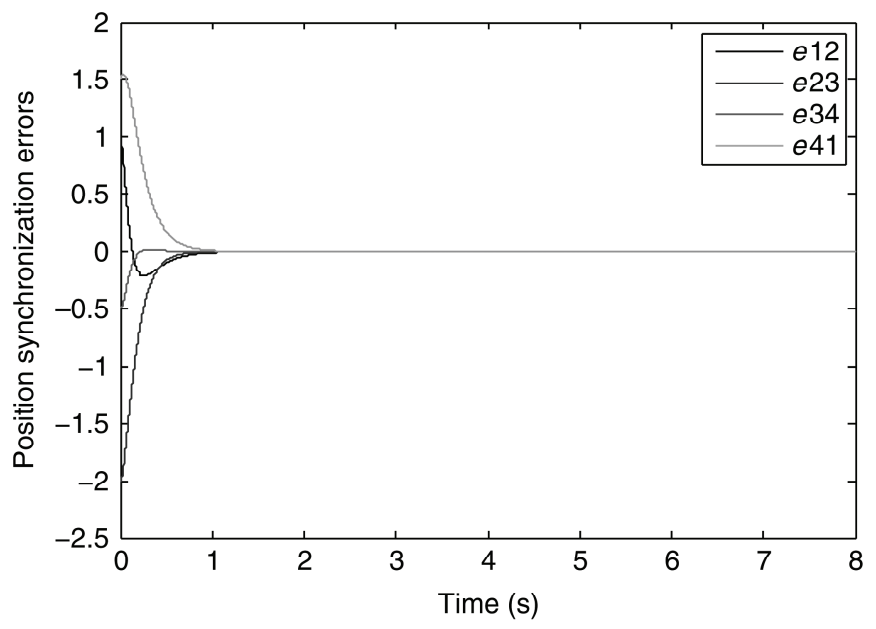

Figure 2. Position-synchronization error.

robots basically converges to zero, which suggests a high synchronization accuracy of the system. When equivalent actuator fault does not occur in the system, the simulation results show that the nominal control law $\tau_{i 0}$ can meet the control requirements.

In the presence of an equivalent actuator fault (i.e., $\left.F_{a i} \neq 0\right)$ a sinusoidal signal $\left[F_{a 1}=1.2 \sin (\pi t)\right]$, a square wave signal with a period of $2 \mathrm{~s}$ and an amplitude of 2 , a cosine signal $\left[F_{a 3}=1.5 \cos (3 t)\right]$, an isosceles triangular wave signal with a period of $2 \mathrm{~s}$ and an amplitude of 1.8 are selected to simulate the actuator fault. Nevertheless, the fault-tolerant control is not performed. That is, the additional control law $\tau_{i f}$ is not used, and the nominal control law $\tau_{i 0}$ is still applied to the control. At this point, Fig. 3 shows the errors $e_{1}, e_{2}, e_{3}$, and $e_{4}$ between the system states $q_{1}, q_{2}, q_{3}$, and $q_{4}$ of four robots and the given input position signals $q_{d}$. Figure 4 shows the synchronization errors $e_{12}, e_{23}, e_{34}$, and $e_{41}$ of the tracking status between adjacent robots. The simulation results are as follows. 


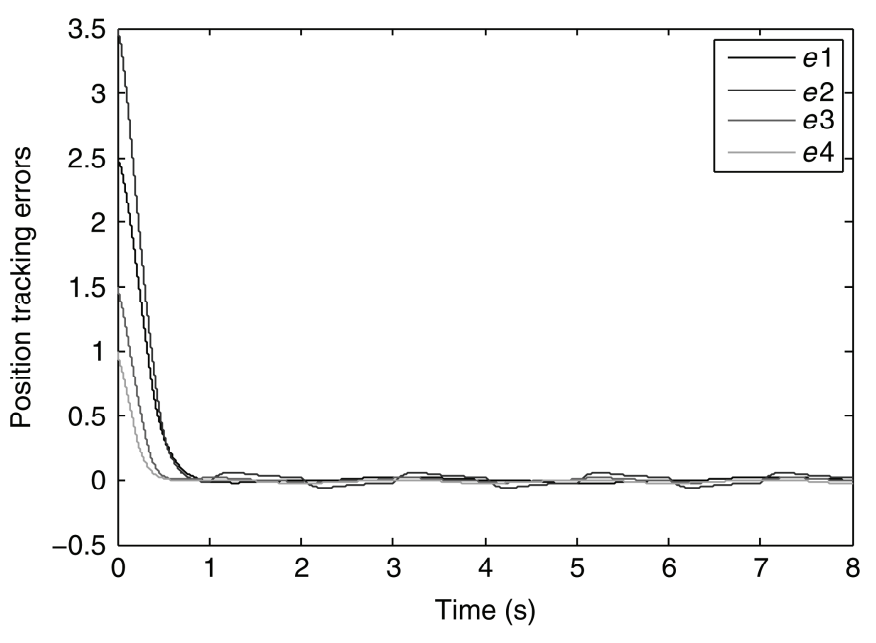

Figure 3. Position-tracking error.

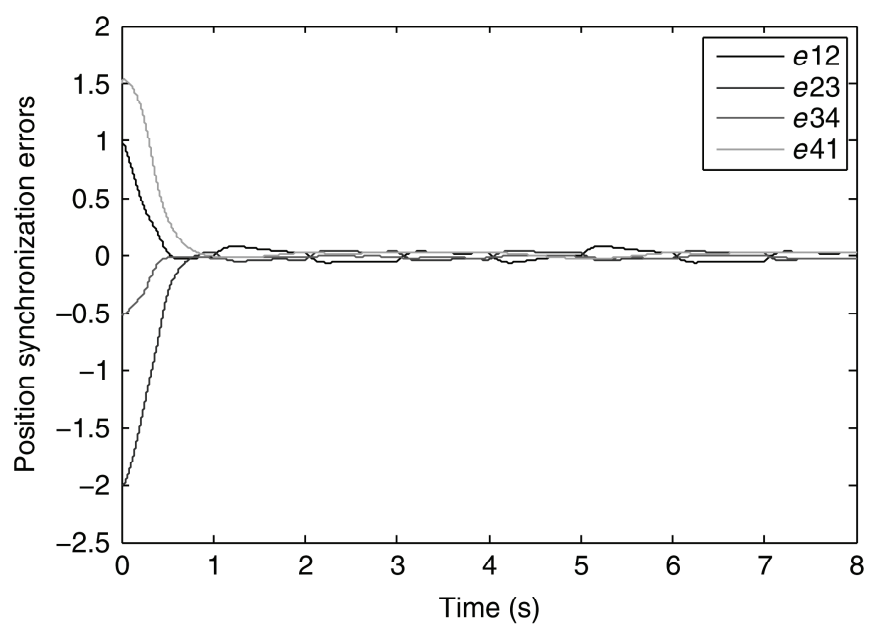

Figure 4. Position-synchronization error.

Figure 3 show unsatisfactory tracking of the given input position signal for the four robots due to certain tracking error. As shown in Fig. 4, the system synchronization accuracy is also unsatisfactory due to certain error. The simulation results show that the nominal control law $\tau_{i 0}$ does not meet the control requirements in the presence of the equivalent actuator fault without fault-tolerant control.

Figures 5-8 present the actual values of the equivalent actuator fault for each individual robot, namely, $F_{a 1}, F_{a 2}$, $F_{a 3}$, and $F_{a 4}$, and the corresponding reconstruction value.

As shown in Figs. 5-8, the reconstructed value of the equivalent actuator fault is basically similar to the actual value. Thus, the reconstruction error is acceptable, and the equivalent actuator fault $F_{a i}$ can be accurately reconstructed using the proposed algorithm.

In the case of fault-tolerant control, by adding the additional control law $\tau_{i f}$, the control law is $\tau_{i}$. Figure 9 shows the errors $e_{1}, e_{2}, e_{3}$, and $e_{4}$ between the system states $q_{1}, q_{2}, q_{3}$, and $q_{4}$ of four robots and the given input position signals $q_{d}$. Figure 10 shows the synchronization errors $e_{12}, e_{23}, e_{34}$, and $e_{41}$ of the tracking status between adjacent robots. The simulation results are as follows.

As shown in Figs. 9 and 10, after adding the additional control law $\tau_{i f}$ for fault-tolerant control, the given input

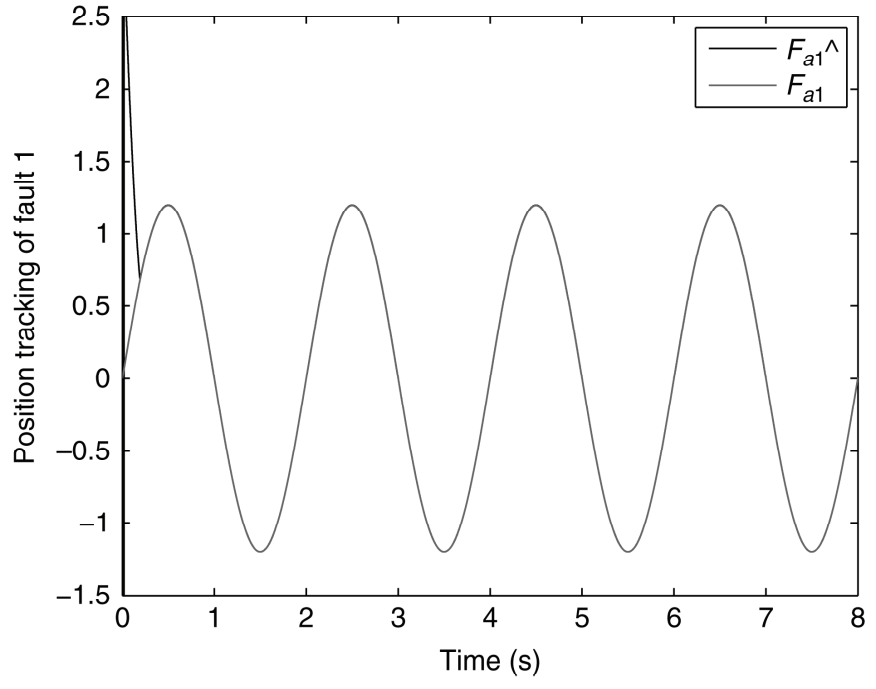

Figure 5. Actual and reconstruction values of equivalent actuator fault $F_{a 1}$.

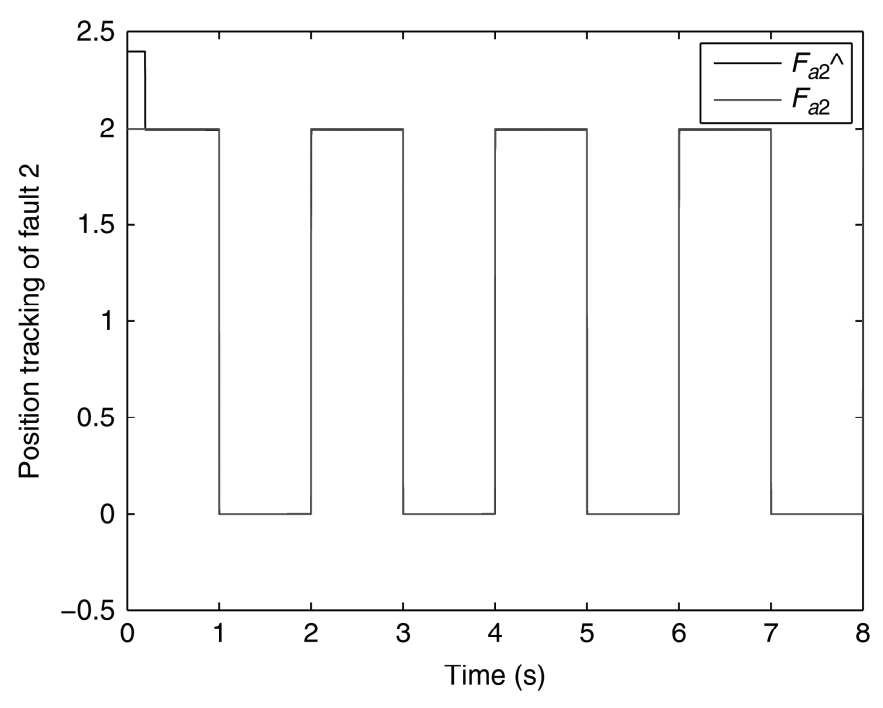

Figure 6. Actual and reconstruction values of equivalent actuator fault $F_{a 2}$.

position signal can be accurately tracked with promising synchronization performance, showing insignificant tracking and synchronization errors. Therefore, the designed control law $\tau_{i}$ can meet the control requirements in the presence of equivalent actuator faults and with fault-tolerant control.

\subsection{Semi-physical Experiment}

A semi-physical experiment platform is established for the multi-robot system, to approximate the whole simulation process to the real engineering environment, better evaluate controller performance, and verify the accuracy and feasibility of the theoretical method proposed in this section.

The entire simulation platform consists of DSP controller, OP5600 simulator, related connected line, and multi-robot system model in the software part and PC monitor interface. 


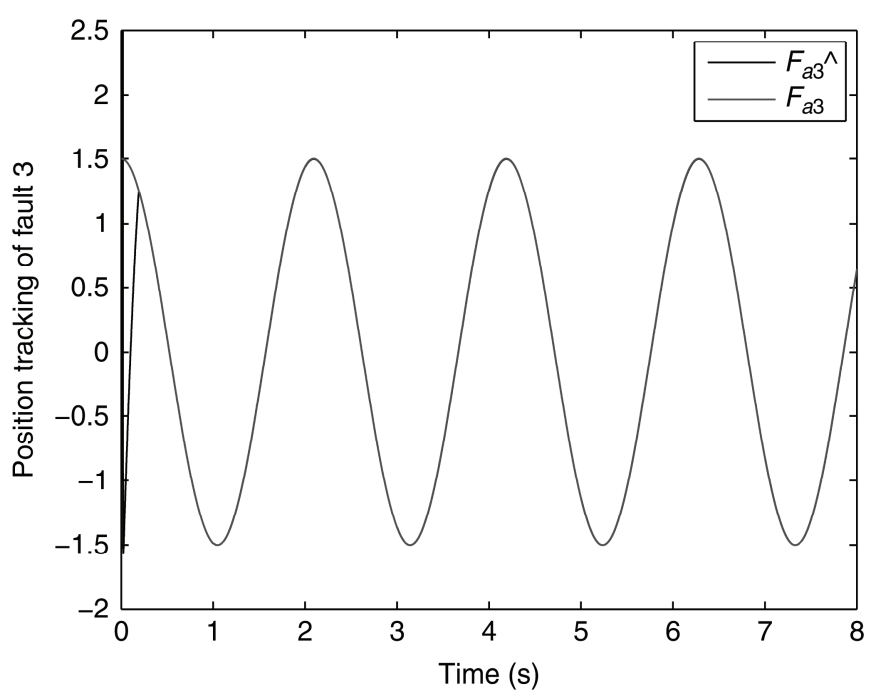

Figure 7. Actual and reconstruction values of equivalent actuator fault $F_{a 3}$.

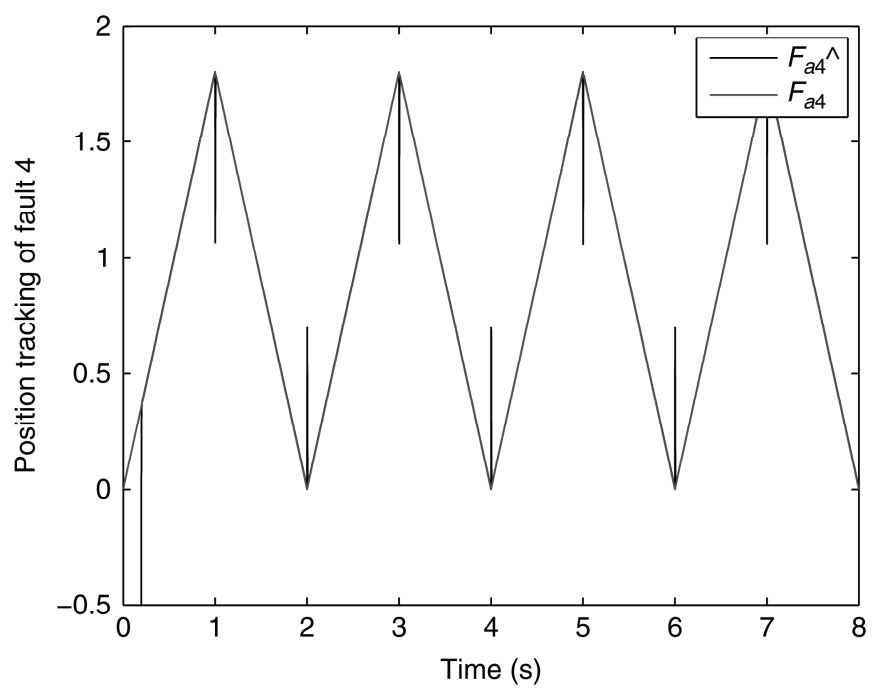

Figure 8. Actual and reconstruction values of equivalent actuator fault $F_{a 4}$.

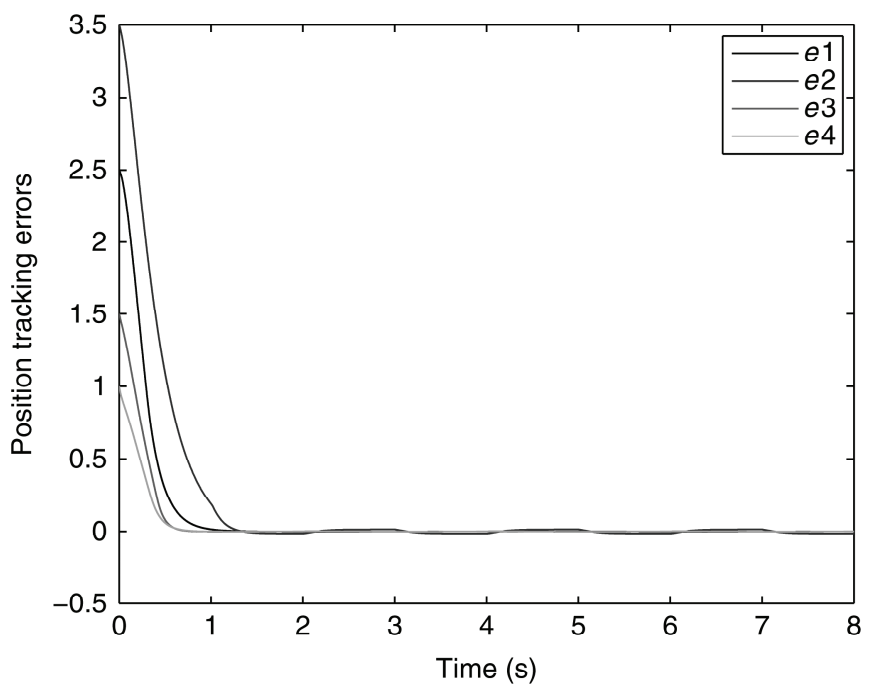

Figure 9. Position-tracking error.

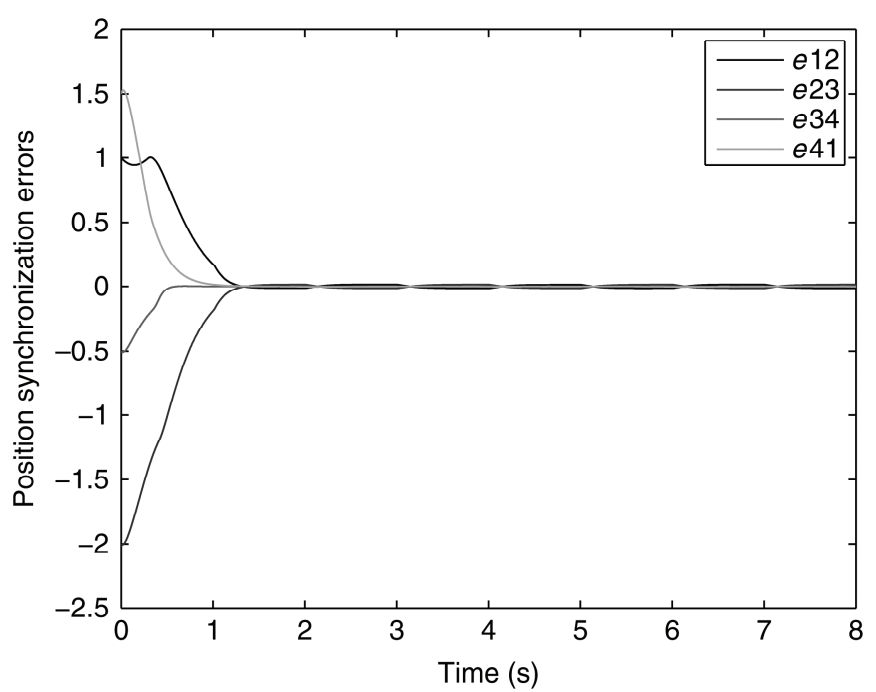

Figure 10. Position-synchronization error.

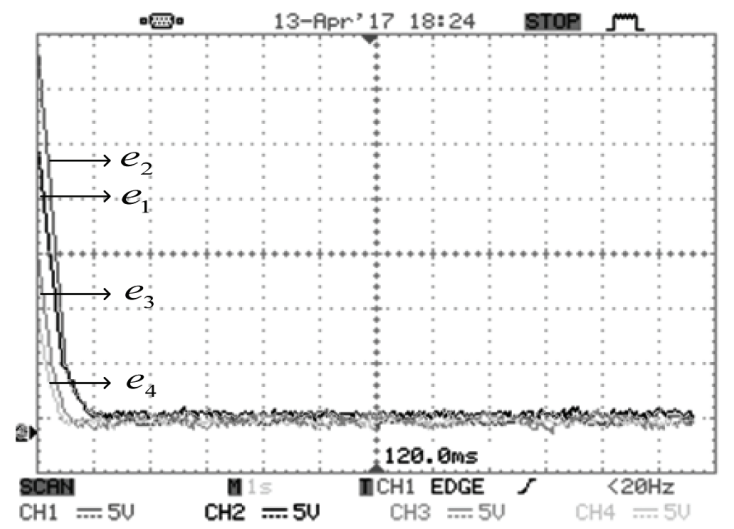

Figure 11. Position-tracking error $\left(e_{i}: 1 /\right.$ unit $)$.

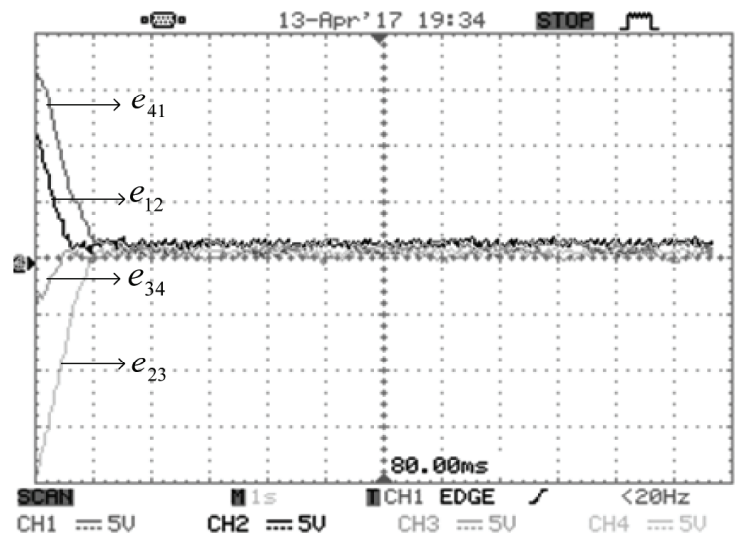

Figure 12. Position-synchronization error $\left(e_{i+1}\right.$ : 1/unit).

In the presence of equivalent actuator faults and without fault-tolerant control, the corresponding tracking and synchronization errors are shown in Figs. 11 and 12 .

As shown in Figs. 11 and 12, the tracking performance and synchronization accuracy of the multi-robot system do not meet the control requirements due to certain errors, which is consistent with the simulation results. 


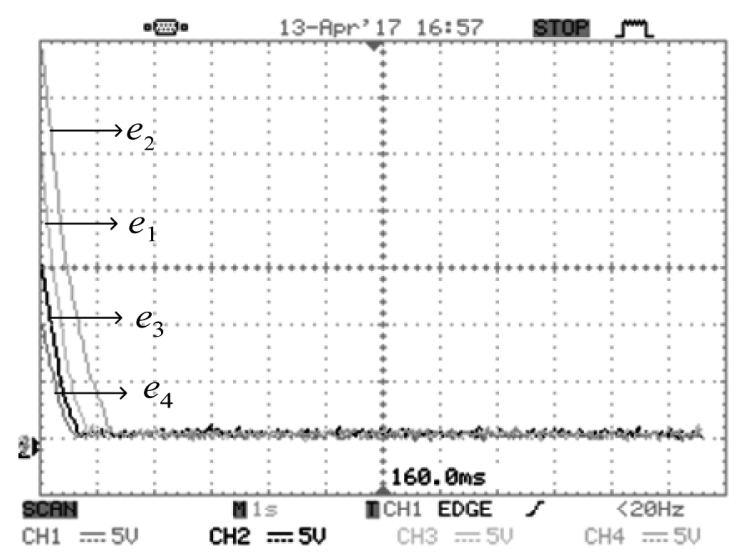

Figure 13. Position-tracking error $\left(e_{i}: 1 /\right.$ unit).

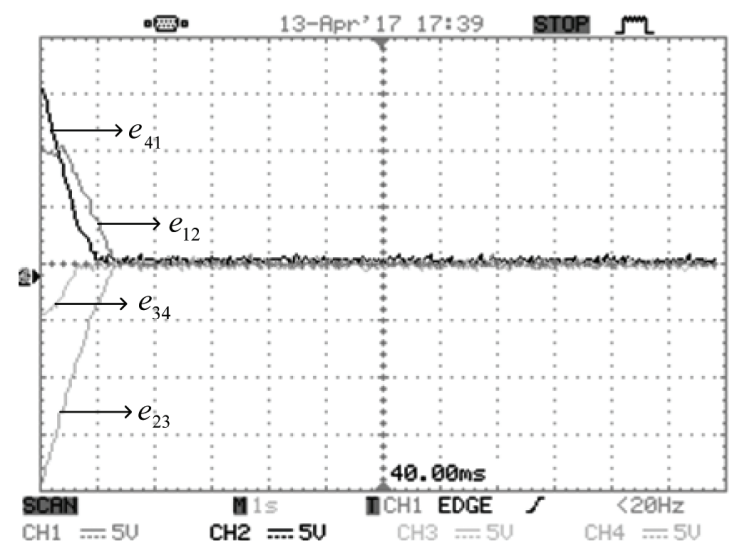

Figure 14. Position-synchronization error $\left(e_{i i+1}: 1 /\right.$ unit).

In the case of fault-tolerant control, the tracking and synchronization errors are shown in Figs. 13 and 14, respectively.

At this point, the tracking and synchronization errors of the multi-robot system are extremely insignificant; thus, the control requirements are satisfied, which is consistent with the simulation results.

\section{Conclusion}

For the problem of synchronous fault-tolerant control for a class of multi-robot systems with actuator faults, a cooperative fault-tolerant control method based on ring coupling has been proposed in this paper. The proposed method can obtain unknown system states, fault information, which can realize online fault-tolerant control and is not limited by the number of controlled objects. Consequently, the multi-robot system has strong robustness and high synchronization accuracy. The simulation and semi-physical experiment results verified the effectiveness of the proposed method. The object considered in this paper is a kind of single DF manipulator, which is not considered in the robot system with multiple DFs and can be further studied in the next step.

\section{Acknowledgments}

This work was supported by the National Key R\&D Program of China (no. 2018YFD0400705), the Natural
Science Foundation of China (nos. 61733004, 61773159, and 61473117), Hunan Provincial Natural Science Foundation of China (nos. 2018JJ2093 and 2017JJ4031), and Key Laboratory for Electric Drive Control and Intelligent Equipment of Hunan Province (no. 2016TP1018).

\section{References}

[1] X. Yi, A. Zhu, S.X. Yang, and C. Luo, A bio-inspired approach to task assignment of swarm robots in 3-D dynamic environments, IEEE Transactions on Cybernetics, 47(4), 2017, 974-983.

[2] J. Ni, X. Yang, J. Chen, and S.X. Yang, Dynamic bioinspired neural network for multi-robot formation control in unknown environments, International Journal of Robotics and Automation, 30(3), 2015, 256-266.

[3] H. Su, M.Z. Chen, X. Wang, and J. Lam, Semiglobal observerbased leader-following consensus with input saturation, IEEE Transactions on Industrial Electronics, 61(6), 2014, 2842-2850.

[4] C.S. Chen, L.Y. Chen, Robust cross-coupling synchronous control by shaping position commands in multiaxes system, IEEE Transactions on Industrial Electronics, 59(12), 2012, 4761-4773.

[5] H. Su, H. Wu, and X. Chen, Observer-based discrete-time nonnegative edge synchronization of networked systems, IEEE Transactions on Neural Networks and Learning Systems, 28(10), 2017, 2446-2455.

[6] A. Zhu and S.X. Yang, An improved approach to dynamic task assignment of non-holonomic multi-robots, International Journal of Robotics and Automation, 26(4), 2011, 362.

[7] G. Chen, Y. Song, and F.L. Lewis, Distributed fault-tolerant control of networked uncertain Euler-Lagrange systems under actuator faults, IEEE Transactions on Cybernetics, $47(7)$, 2017, 1706-1718.

[8] D. Ye, X. Zhao, and B. Cao, Distributed adaptive faulttolerant consensus tracking of multi-agent systems against timevarying actuator faults, IET Control Theory Appl, 10(5), 2016, $554-563$.

[9] M. Khalili, X. Zhang, M.M. Polycarpou, and T. Parisini, Distributed adaptive fault-tolerant control of uncertain multiagent systems, Automatica, 87, 2018, 142-151.

[10] Y. Ai, B. Pan, G. Niu, Y. Fu, and S. Wang, Master-slave control technology of isomeric surgical robot for minimally invasive surgery, 2016 IEEE International Conference on Robotics and Biomimetics (ROBIO), Qingdao, China, 2016, 2134-2139.

[11] W. Chen, Y. Wu, R. Du, Q. Chen, and X. Wu, Speed tracking and synchronization of a dual-motor system via second order sliding mode control, Mathematical Problems in Engineering, 2013, Article ID 919837, 2013, 10. doi:10.1155/2013/919837.

[12] J. Andert, S. Klein, R. Savelsberg, S. Pischinger, and K. Hameyer, Virtual shaft: Synchronized motion control for real time testing of automotive powertrains, Control Engineering Practice, 56, 2016, 101-110.

[13] B. Liu, D.J. Hill, and T. Liu, Exponential input-to-state stability under events for hybrid dynamical networks with coupling time-delays, Journal of the Franklin Institute, 354, 2017, 7476-7503.

[14] B. Liu, T. Liu, and C.X. Dou, Stability of discrete-time delayed impulsive systems with application to multi-tracking, International Journal of Control, 87(5), 2014, 911-924.

[15] B. Liu and D.J. Hill, Impulsive consensus for complex dynamical networks with nonidentical nodes and coupling time-delays, SIAM Journal on Control and Optimization, 49(2), 2011, 315-338.

[16] N.G. Ulu, E. Ulu, and M. Cakmakci, Design and analysis of a modular learning based cross-coupled control algorithm for multi-axis precision positioning systems, International Journal of Control, Automation, and Systems, 14(1), 2016, 272-281.

[17] J. Li, Y. Fang, X. Huang, and J. Li, Comparison of synchronization control techniques for traction motors of high-speed trains, Proceedings of the International Conference on Electrical Machines and Systems, IEEE, Hangzhou, China, 2014, 2114-2119. 
[18] H.H. Shi, X. Wu, L. Hua, H.G. Ji, The simulation research of synchronization control based on the virtual shaft control and the relative coupling control, Advanced Materials Research, 1049, 2014, 1111-1115.

[19] G. Liu, W. Xu, and J. Sun, PMSM adaptive backstepping synchronization control based on improved deviation coupling, Small Special Electrical Machines, 2, 44(2), 2016, 45-49.

[20] L.B. Li and L.L. Sun, Multi-channel synchronization control based on mean of deviation coupling control, OptikInternational Journal for Light and Electron Optics, 127(8), 2016, 3703-3707.

[21] R. Liu, J.Z. Sun, Y.Q. Luo, W. Sun, and W.D. Li, Research on multi-motor synchronization control based on the ring coupling strategy for cutterhead driving system of shield machines, Applied Mechanics and Materials. Trans Tech Publications, 52, 2011, 65-72.

[22] L.B. Li, L.L. Sun, S.Z. Zhang, and Q.Q. Yang, Speed tracking and synchronization of multiple motors using ring coupling control and adaptive sliding mode control, ISA Transactions, $58,2015,635-649$.

[23] M.R. Soltanpour, B. Zolfaghari, M. Soltani, and M.H Khooban, Fuzzy sliding mode control design for a class of nonlinear systems with structured and unstructured uncertainties, International Journal of Innovative Computing, Information and Control, 19(7), 2013, 2713-2726.

\section{Biographies}

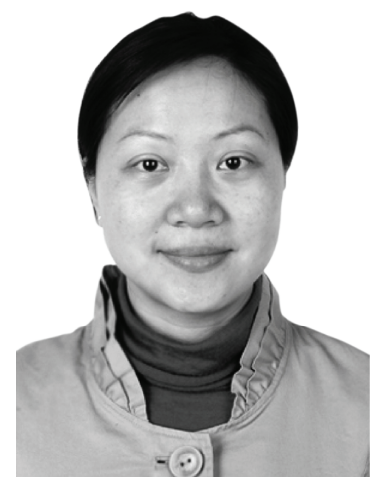

Jing $\mathrm{He}$ received her M.S. degree in computer engineering from the Central South University of Forestry and Technology, Changsha, China, in 2002 and the Ph.D. degree in mechatronics engineering from the National University of Defense Technology, Changsha, in 2009. She was a Visiting Research Fellow with Helsinki University of Technology, Helsinki, Finland, from September 2004 to November 2005; the University of Waterloo, Waterloo, ON, Canada, from May 2007 to April 2008; Tokyo University of Technology, Tokyo, Japan, from March to May 2016; and the University of Alabama, Tuscaloosa, AL, USA, from January 2017 to December 2017. Since September 1992, she has been with the College of Electrical and Information Engineering, Hunan University of Technology, Zhuzhou, China, where she has been a Professor since 2009. Her main research direction is electro-mechanical system fault diagnosis.

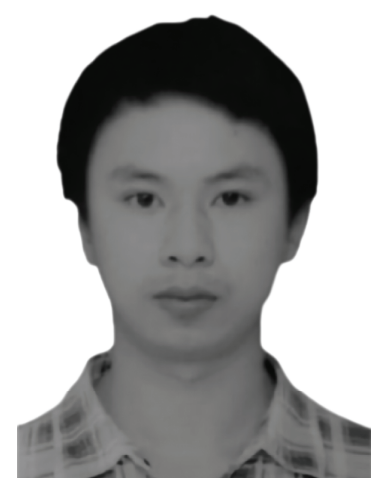

Lin $M i$ received his B.Sc. degree from Hunan University of Technology in 2014, and the M.S. degree in Control Theory and Control Engineering from Hunan University of Technology, China, in 2017. His main research direction is fault reconstruction on non-linear systems and sliding mode control.

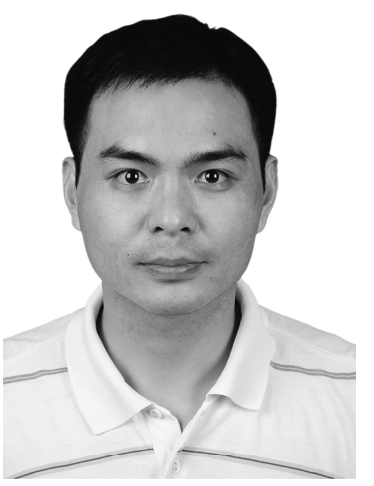

Jianhua Liu received his B.S. degree from Xiangtan University, China, in 2004, and the M.S. and Ph.D. degrees in control engineering and science from Central South University, China, in 2009 and 2013, respectively. Since 2013, he has been with the College of Electrical and Information Engineering, Hunan University of Technology, China. His main research direction is electromechanical system fault diagnosis.

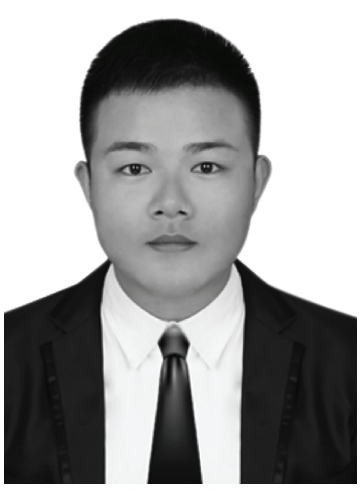

Xiang Cheng received his B.Sc. degree from Nanyang Institute of Technology in 2014. Now, he is an M.Sc. candidate in Hunan University of Technology. His main research direction is power transmission and fault diagnosis.

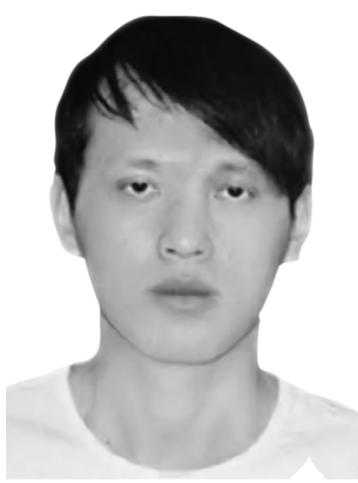

Zhenzhen Lin received his B.S. degree in physics from Liaoning University, Shenyang, China, in 2014. He is currently pursuing the M.S. degree with the College of Electrical and Information Engineering, Hunan University of Technology, Zhuzhou, China. His main research direction is fault diagnosis on electric machines and industrial process control.

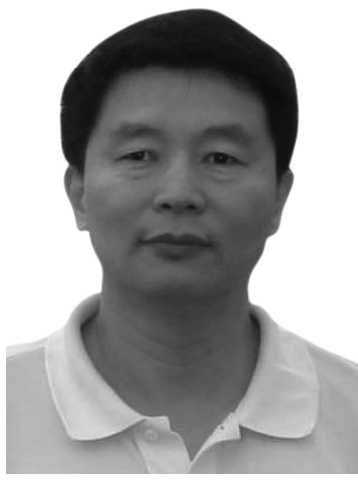

Changfan Zhang received his M.S. degree in electronics engineering from Southwest Jiaotong University, Chengdu, China, in 1989 and the Ph.D. degree in control theory and engineering from Hunan University, Changsha, China, in 2001. He was a Postdoctoral Fellow with Central South University, Changsha, from October 2001 to October 2003, and a Visiting Research Fellow with the University of Waterloo, Waterloo, ON, Canada, from May 2007 to April 2008. Since February 1982, he has been with the College of Electrical and Information Engineering, Hunan University of Technology, Zhuzhou, China, where he has been a Professor since 2001. His main research direction is non-linear control and applications. 